\title{
Preisverleihung der mathematischen Adventskalender
}

\author{
David Vogel
}

Für manche dürfte es Premiere gewesen sein, für andere ein zweites Weihnachten. Aber kaum betraten die jungen Gewinnerinnen und Gewinner der Mathematikwettbewerbe „Mathe im Advent" und „Matheon-Kalender" die Bühne, verflog ihr Lampenfieber. Dafür sorgten die Applauskulisse im Saal und die Vorfreude auf Tablets, Spielkonsolen und Bücher als Preise.

Im Dezember bewiesen sich rund 117000 junge Mathefans täglich an Aufgaben in Form von illustrierten Wichtelgeschichten, die in drei Kalendern für die Klassenstufen 4-6, 7-9 und 1o+ auf mathekalender.de angeboten wurden. Nun, am 20. Januar, belebten rund sechshundert der erfolgreichsten unter ihnen das Audimax der TU Berlin, um Gruppe für Gruppe geehrt zu werden.

Mit dabei: die Klasse 3 a von der Paul-Klee-Schule in Köln. Auch wenn sie die Aufgaben und die darin agie- renden Helden aus dem Weihnachtsdorf schnell liebgewonnen hatten - sie zu lösen war für die Jüngsten noch eine ziemliche Hürde. Das weiß niemand besser als Klassenlehrerin Julia Froetsch, die aus „Mathe im Advent" fächerübergreifendes Kapital schlug. Nutzte sie die Wichtelgeschichten doch zunächst als Leseübungen, damit ihre Schülerinnen und Schüler die Aufgaben präzise genug verstehen. Und auch ihre Lösungen mussten die Kinder ordentlich begründen. Mit Erfolg: Die Kölner gewannen als „Frühstarter“. Und im Bann der Wichtelwelt wurden sie sogar kreativ. Da fingen in der Phantasie Geschenke an, vom Schlitten zu purzeln (siehe Seite 52) und flugs war eine Serie eigener Matheaufgaben samt Zeichnungen verfasst, die die eifrigen Frühstarter dem Team von „Mathe im Advent" auf der Bühne schenkten.
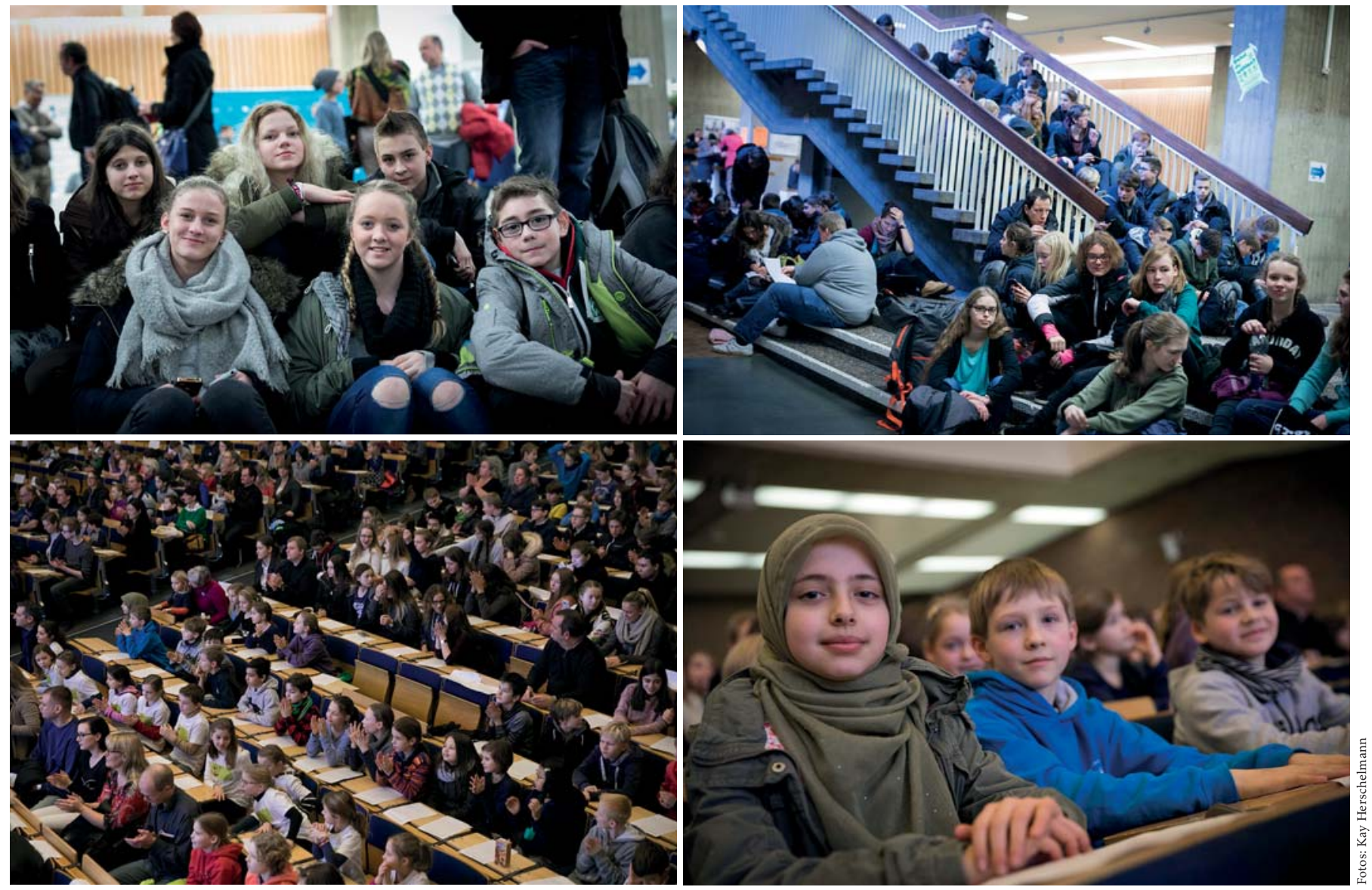
Während „Mathe im Advent“ eine möglichst breite Schülerschaft der Unter- und Mittelstufe spielerisch für die Mathematik begeistern will, setzt der MatheonKalender auf Talentförderung in den oberen Klassen. Hier dominieren Cracks wie Martin Drees aus Cadolzburg, der keine Gelegenheit auslässt, sein Können zu erproben und zu perfektionieren. Nur Schulhausaufgaben schenke er sich, gesteht er, die Urkunde unterm Arm. Recht dazu geben ihm seine Erfolge bei der Kopfrechenmeisterschaft, den „Mathe-Olympiaden“ und dem „KänguruWettbewerb“, die Trainings im Schachverein, dem „QEDVerein“, bei „Jugend trainiert Mathematik“. „Nun also Matheon-Sieger“, setzt er lässig fort. „Momentan mache ich viel Wettberbsmathematik, aber nicht mehr lange. Ich werde wohl bald Mathe studieren in Bonn." Womit sich die zweite Parallele zwischen dem Sieger von 2016 und einem anderen jungen Talent ergäbe: Ein gewisser Peter Scholze rechnete sich beim Matheon-Kalender auf Platz 1, damals vor seinem Senkrechtstart. 2012 wurde er zum jüngsten Mathematikprofessor Deutschlands nach Bonn berufen. Auch Drees wirkt dem Kalender schon jetzt entwachsen: „Dieses Mal war nur eine wirklich schwere Aufgabe dabei!“ Lösen Sie Marc Uetz' „Crossnumbers“ auch in unter zehn Minuten?

In punkto Breitenwirkung geht der Pokal nach BadenWürttemberg. 806 Schülerinnen und Schüler konnte das
Crossnumbers (Marc Uetz, Universiteit Twente)

Every empty square in the following diagram should be filled in with a digit; a digit may be used multiple times. In this puzzle, each of the four elves Alphonso, Bartolomeo, Cristiano and Domingo is at least 10 years old.

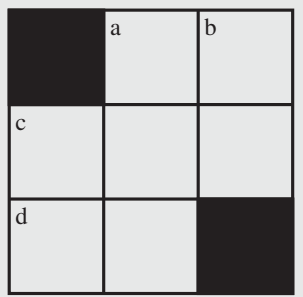

Horizontal:

a. Alphonso's age. c. Sum of the ages from three of the four elves. d. Domingo's age.

Vertical:

a. Sum of the ages of all four elves. b. Bartolomeo's age. c. Cristiano's age.

Question: Which digit appears more than once in the solution?

Possible answers:

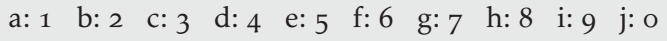

Friedrich-Schiller-Gymnasium aus Marbach zur Teilnahme an „Mathe im Advent“ motivieren, so viel wie keine andere Schule. Den beträchtlichen Zulauf initiierte das Lehrerehepaar Sylvia und Thomas Tressel per Dominoeffekt: Erst überzeugten sie 19 Lehrerkollegen, daraufhin
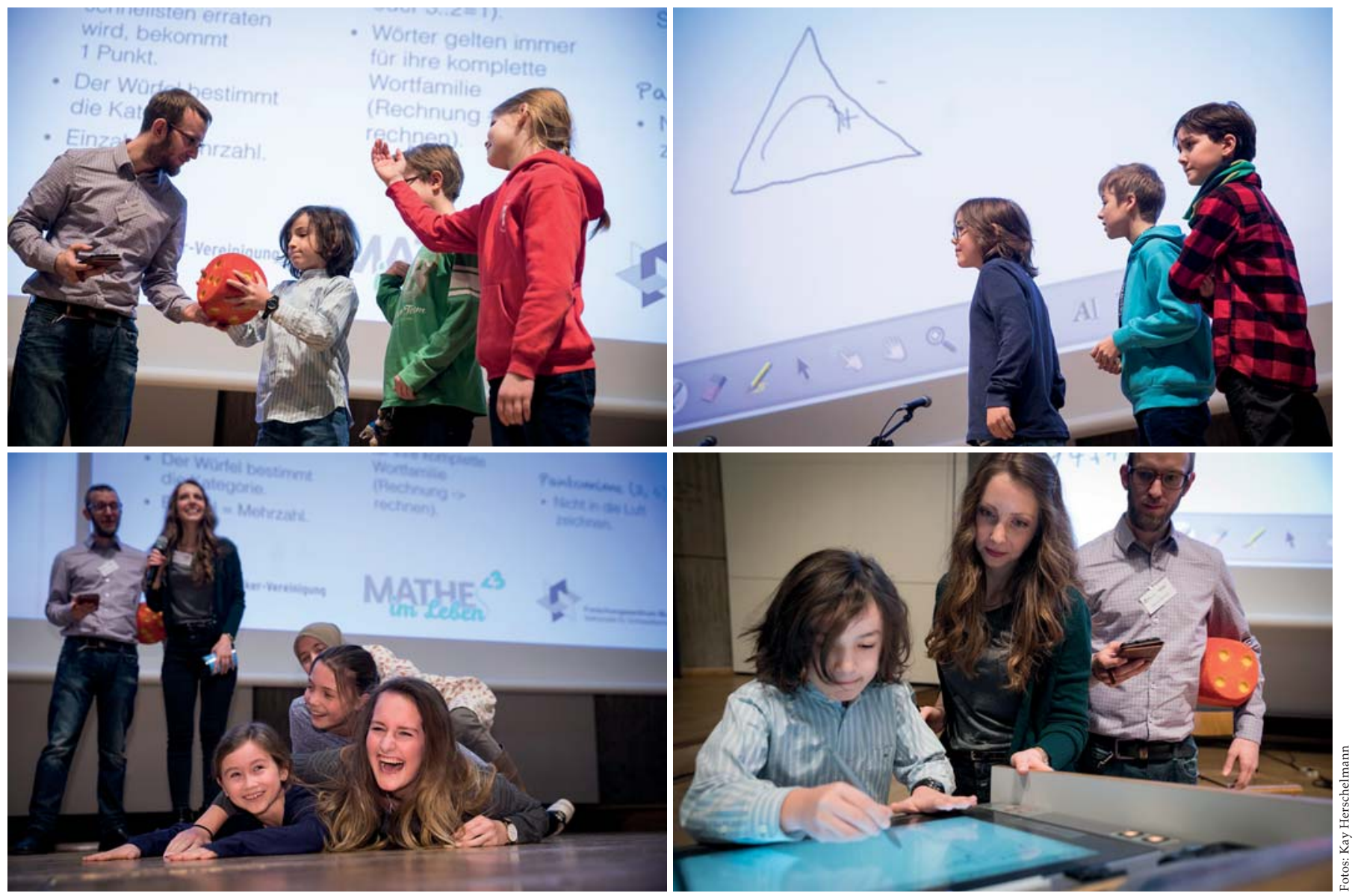


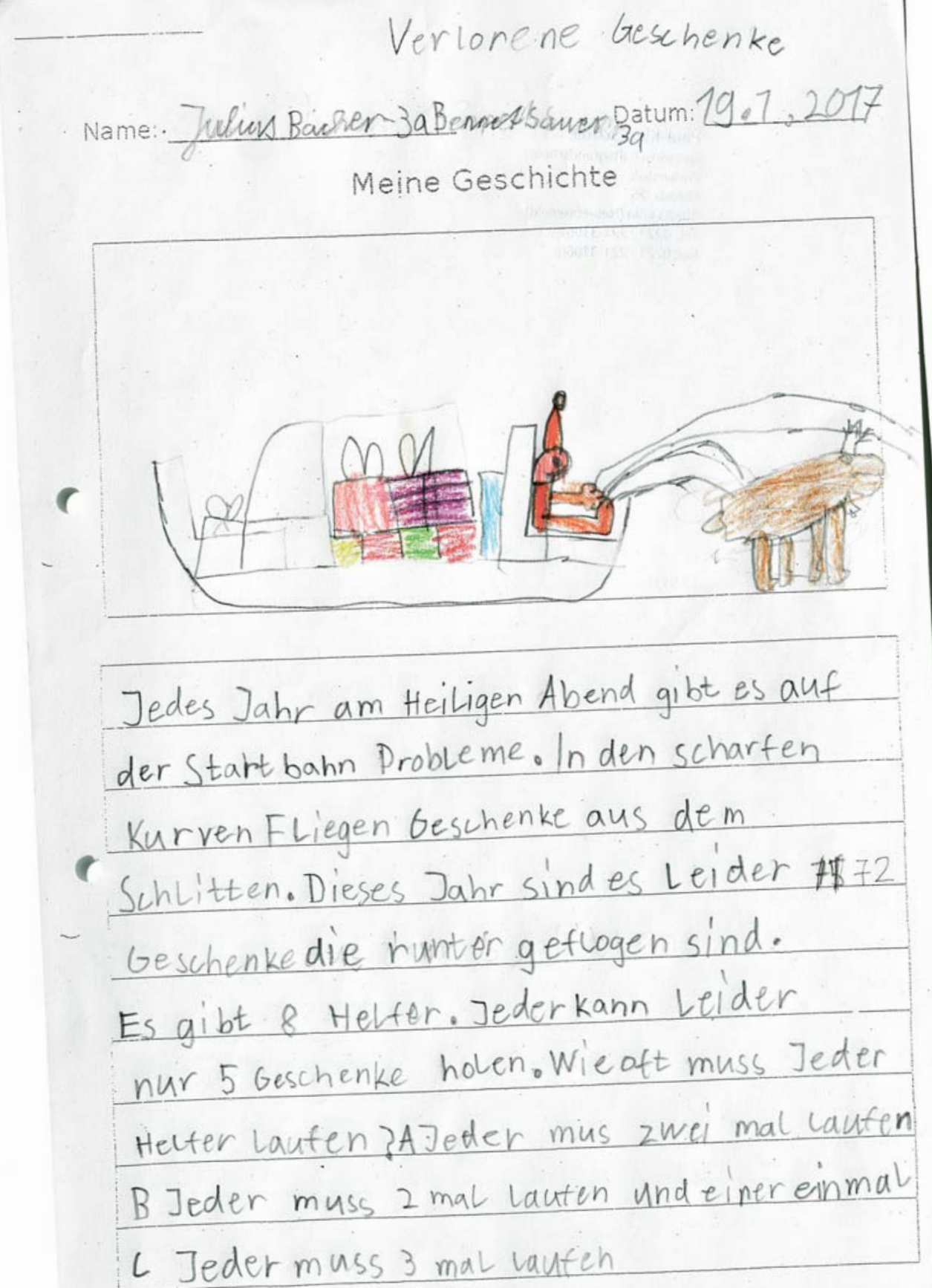

zogen Schüler dutzendfach mit. „In den großen Pausen haben sich die Schüler über die Aufgaben unterhalten und teilweise heftig über die mathematischen Inhalte diskutiert", schildern die Tressels die Begeisterungswelle. Für die gelungene Lobbyarbeit an der Basis wurden sie von der DMV als Mathemacher im Januar ausgezeichnet [siehe Seite 44]. Ihr Credo: „Wir sind überzeugt davon, dass die Aufgaben die Motivation für das Fach Mathematik erhöhen und auch das logisch-mathematische Denken schulen." 